\title{
Potential effectiveness of Community Health Strategy to promote exclusive breastfeeding in urban poor settings in Nairobi, Kenya: a quasi-experimental study
}

\author{
E. W. Kimani-Murage ${ }^{1,2 *}$, S. A. Norris ${ }^{3}$, M. K. Mutua ${ }^{1}$, F. Wekesah ${ }^{1}$, M. Wanjohi ${ }^{1}$, N. Muhia ${ }^{1}$, P. Muriuki $^{1}$, T. Egondi ${ }^{1}$, \\ C. Kyobutungi ${ }^{1}$, A. C. Ezeh $^{1}$, R. N. Musoke ${ }^{4}$, S. T. McGarvey ${ }^{2}$, N. J. Madise ${ }^{5}$ and P. L. Griffiths ${ }^{3,6}$ \\ ${ }^{1}$ African Population and Health Research Center (APHRC), Nairobi, Kenya \\ ${ }^{2}$ International Health Institute, Brown University, Providence, RI, USA \\ ${ }^{3}$ MRC/Wits Developmental Pathways for Health Research Unit, Faculty of Health Sciences, University of the Witwatersrand, Johannesburg, South Africa \\ ${ }^{4}$ Department of Paediatrics, University of Nairobi, Nairobi, Kenya \\ ${ }^{5}$ Centre for Global Health, Population, Poverty, and Policy University of Southampton, Southampton, UK \\ ${ }^{6}$ Centre for Global Health and Human Development, Loughborough University, Loughborough, UK
}

Early nutrition is critical for later health and sustainable development. We determined potential effectiveness of the Kenyan Community Health Strategy in promoting exclusive breastfeeding (EBF) in urban poor settings in Nairobi, Kenya. We used a quasi-experimental study design, based on three studies [Pre-intervention (2007-2011; $n=5824)$, Intervention (2012-2015; $n=1110)$ and Comparison (2012-2014; $n=487)$ ], which followed mother-child pairs longitudinally to establish EBF rates from 0 to 6 months. The Maternal, Infant and Young Child Nutrition (MIYCN) study was a cluster randomized trial; the control arm (MIYCN-Control) received standard care involving community health workers (CHWs) visits for counselling on antenatal and postnatal care. The intervention arm (MIYCN-Intervention) received standard care and regular MIYCN counselling by trained CHWs. Both groups received MIYCN information materials. We tested differences in EBF rates from 0 to 6 months among four study groups (Pre-intervention, MIYCN-Intervention, MIYCN-Control and Comparison) using a $\chi^{2}$ test and logistic regression. At 6 months, the prevalence of EBF was $2 \%$ in the Pre-intervention group compared with 55\% in the MIYCN-Intervention group, $55 \%$ in the MIYCN-Control group and 3\% in the Comparison group $(P<0.05)$. After adjusting for baseline characteristics, the odds ratio for EBF from birth to 6 months was 66.9 (95\% CI 45.4-96.4), 84.3 (95\% CI 40.7-174.6) and 3.9 (95\% CI 1.8-8.4) for the MIYCN-Intervention, MIYCN-Control and Comparison group, respectively, compared with the Pre-intervention group. There is potential effectiveness of the Kenya national Community Health Strategy in promoting EBF in urban poor settings where health care access is limited.

Received 13 July 2015; Revised 20 October 2015; Accepted 12 November 2015; First published online 28 December 2015

Key words: Community health workers, exclusive breastfeeding, Kenya, sub-Saharan Africa, Urban slums

\section{Background}

Promotion of breastfeeding is considered one of the high impact nutrition interventions. ${ }^{1,2}$ Implementing interventions which promote breastfeeding could prevent about $13 \%$ of under-five deaths in countries with high mortality rates. ${ }^{3}$ Breastfeeding confers both short-term and long-term benefits to the child: it reduces infections and mortality among infants; improves mental and motor development; and protects against obesity and metabolic diseases later in the life course. ${ }^{3-7}$ The World Health Organization (WHO) ${ }^{8}$ recommends exclusive breastfeeding $(\mathrm{EBF})$ in the first 6 months of life followed by extended breastfeeding for 2 years or beyond for optimal growth, development and survival of the child. Non-EBF in the first 6 months has been associated with higher morbidity and

*Address for correspondence: E. W. Kimani-Murage, African Population and Health Research Center (APHRC), P.O. 10787, 00100 Nairobi, Kenya. (Email ekimani@aphrc.org) mortality compared with EBF, and likewise, no breastfeeding at all has been associated with higher risk of morbidity and mortality in children 6-23 months compared with breastfeeding. ${ }^{9,10}$ Recent evidence by Victora et al. ${ }^{11}$ based on a prospective, population-based birth cohort study launched in 1982 in Pelotas, Brazil further indicates that the duration of total breastfeeding improves intelligence quotient, educational attainment and income in adulthood.

Strategies for the promotion of breastfeeding have been defined and implemented in various settings, including the Baby Friendly Hospital Initiative (BFHI), a global strategy which promotes breastfeeding in maternity wards around the time of delivery. The effectiveness of the BFHI in promoting optimal breastfeeding practices has been established, particularly in more developed countries where health care is accessible, hence health facility deliveries prevalent. ${ }^{12-15}$ For example, the Promotion of Breastfeeding Intervention Trial study in the Republic of Belarus found that the BFHI 
was effective in improving both duration and exclusivity of breastfeeding. ${ }^{12,13}$ However, in low-income countries (LICs), where many deliveries do not occur in health facilities, ${ }^{16}$ the effectiveness of the BFHI may be limited.

In Kenya, the situation of infant and young child-feeding (IYCF) practices has been poor, with the prevalence of EBF for children aged 0-6 months (using 24-hour recall) at only 13\% in 2003 , improving to $32 \%$ in $2008 .{ }^{17,18}$ The situation is very poor in urban poor settings where barely $2 \%$ of children are exclusively breastfed cumulatively from birth to 6 months. ${ }^{19}$ To improve the situation in Kenya, the Ministry of Health in Kenya developed an infant and young child nutrition (IYCN) strategy in 2007 (IYCN strategy 2007-2010), aimed at promoting optimal IYCN practices in the country. ${ }^{8,20}$ The strategy was actualized through revitalization of the BFHI, which has been in existence in Kenya but hardly functional since the $1980 \mathrm{~s}^{21}$ Anecdotal evidence indicates that only slightly over 10 hospitals in Kenya are certified as baby friendly. Further, realizing the need to extend the counselling and support to the community level, given that many births have been occurring at home, ${ }^{17,18}$ the Ministry of Health has proposed the adoption of the Baby Friendly Community Initiative (BFCI), a global initiative that extends the principles of BFHI to the community level, as outlined in the 2012-2017 Nutrition Action Plan (http://bit.ly/1G8RVz8). The BFCI therefore complements the BFHI by extending counselling on Maternal, Infant and Young Child Nutrition (MIYCN) to the community level as proposed in the current (2012-2017) MIYCN strategy. In line with this, the new national MIYCN strategy (2012-2017) recognizes the important role of community health workers (CHWs), currently referred to as community health volunteers in Kenya, in promotion of optimal MIYCN practices. It is expected that the CHWs would promote MIYCN through the Community Health Strategy, a government initiative that aims at using CHWs to promote health in the community. ${ }^{22}$ However, achievement of this aspect of the MIYCN strategy is hampered by the fact that optimal implementation of the proposed national Community Health Strategy in Kenya is barred by limited funding. The Community Health Strategy was rolled out in Kenya in 2007 although it is not yet fully functional in many parts of the country. ${ }^{23}$

To inform potential effectiveness of using CHWs to promote EBF and other optimal IYCF practices in urban poor settings in Kenya where access to quality health care is limited, we designed a cluster randomized controlled trial (CRT) in urban poor settings in Nairobi, Kenya. We used CHWs within the Community Health Strategy to offer personalized home-based counselling on optimal infant-feeding practices. ${ }^{24}$ CHWs in the intervention arm were trained to offer counselling on MIYCN while those in the control arm were trained to offer standard care including counselling on antenatal and postnatal care. In both arms, CHWs were given financial incentives and were supervised routinely. In addition, women received information, education and communication (IEC) materials on MIYCN, distributed by the CHWs in both groups. The primary aim of the CRT was to determine the effectiveness of the intervention on improving EBF in the first 6 months. Preliminary analyses (not shown) have revealed no statistically significant differences in the rate of EBF from birth to 6 months between the intervention and the control group, despite the rates increasing from $-2 \%$ at baseline to over $50 \%$ in both groups following the intervention. We argue that, it was likely that the intervention was actually effective in increasing the rate of EBF, but we were unable to show this difference due to potential secular trends and/or contamination of the control group by inadvertent breastfeeding information, among other possibilities (Kimani-Murage et al., unpublished). In this paper, we use other contextual data to determine whether the intervention did potentially work to improve EBF rates, over and above secular trends. In other words, we aim to determine potential effectiveness of the national Community Health Strategy involving home-based counselling visits by CHWs on EBF. We hypothesize that EBF for 6 months increased significantly in households receiving regular $\mathrm{CHW}$ counselling visits in our study regardless of study arm, that is, either intervention and control arms, but little change occurred in households, which were not in our study as these households were unlikely to receive regular CHW visits.

\section{Methods \\ Study setting}

The study was carried out in two slums of Nairobi, Kenya (Korogocho and Viwandani) where the African Population and Health Research Center (APHRC) runs the Nairobi Urban Health and Demographic Surveillance System (NUHDSS), covering close to 70,000 residents. The NUHDSS involves a systematic quarterly recording of vital demographic events including births, deaths and migrations occurring among residents of all households in the NUHDSS area since 2003. Other data including household assets, morbidity and education are also collected and updated approximately once a year through NUHDSS. The two slums are located about $7 \mathrm{~km}$ from each other. They are densely populated with 63,318 and 52,583 inhabitants $/ \mathrm{km}^{2}$, respectively, and are characterized by poor housing, lack of basic infrastructure, violence, insecurity, high unemployment rates and poverty, and poor health indicators. ${ }^{25-29}$ Poor breastfeeding and other infant-feeding practices have been documented in the study setting, and these have been attributed to poor knowledge, lack of professional health support to mothers, food insecurity and women's occupation that are incompatible with EBF. ${ }^{19,30}$ High levels of food insecurity have been documented in these areas, with over $80 \%$ of the households either severely, moderately or mildly food insecure, in terms of household's food access, measured using the household food insecurity access score. ${ }^{31}$ Undernutrition is high, with stunting at $45 \%$ for children aged $<5$ years $^{32}$ and is strongly associated with food poverty and other forms of poverty (assets and subjective poverty). ${ }^{33}$ 
Unsurprisingly, high under-five child mortality has been documented, at 79 deaths/1000 live births, higher than among other population groups in Kenya including rural settings. ${ }^{27}$ More information regarding the study area and the NUHDSS can be obtained from a previous publication. ${ }^{34}$

\section{Data}

We use data from three studies: a longitudinal observational study conducted between 2007 and 2011, herein referred to as the Pre-intervention study; a cluster randomized study conducted between 2012 and 2015, herein referred to as the MIYCN Intervention study; and a longitudinal study conducted between 20012 and 2014, herein referred to as the Comparison study.

\section{Pre-intervention study}

Details about the pre-intervention study procedures are published elsewhere. ${ }^{19,33}$ We use data from the maternal and child health $(\mathrm{MCH})$ component of a broader longitudinal study entitled Urbanization, Poverty and Health Dynamics (UPHD) in Sub-Saharan Africa, funded by the Wellcome Trust, and nested within the NUHDSS. The UPHD study addressed key health consequences of rapid urbanization and growing urban poverty at different stages of the life course namely childhood, adolescence, adulthood and old age. The UPHD study was conducted between 2007 and 2010, but the MCH component was extended beyond 2010 through another project referred to us INDEPTH Vaccination Study (IVP), funded by the Danish Development Agency through the INDEPTH network.

Data included in the analysis were collected between February 2007 and December 2011. All women who were resident in the NUHDSS sites who gave birth from September 2006 to December 2011 and their children were enrolled in the study. Efforts were made to recruit the mother-child pairs as early as possible after birth. The mother-child pairs were followed up until the child was 5 years. Data were collected at recruitment and updated every 4 months during the follow-up period. Questions on breastfeeding were asked during the 1st year of life. During each visit field interviewers administered questionnaires to collect data on breastfeeding and other feeding practices, vaccination, health care seeking and health status. Anthropometric measurements were also carried out.

\section{Intervention study}

The study protocol is already published. ${ }^{24}$ Here we only detail methods relevant to the research question for this paper.

The study, funded by the Wellcome Trust, was a randomized controlled trial using cluster randomization, ${ }^{35,36}$ conducted between September 2012 and February 2015. For pragmatic purposes, Community Units (CUs), defined by the national Community Health Strategy ${ }^{22}$ were used as clusters. CUs are geographically defined units with an approximate population of 5000 people. Within each CU, a CHW provides primary health care services to people. Where the CUs did not exist, APHRC facilitated set-up of the CUs by the government. Cluster randomization was preferred over individual-level randomization to minimize contamination, given the nature of the intervention as described below and for pragmatic purposes in case of future scale-up of the intervention within the Kenyan health system.

The inclusion criteria included all pregnant women aged between 12 and 49 years old, who were resident within the defined study area and their respective babies (when born). The exclusion criteria included (a) women of reproductive age who gave birth before receiving the intervention. Although the reproductive age in most studies is usually defined as 15-49 years, we included girls aged $12-14$ years in our criteria as a substantial proportion (close to 10\%) of adolescents in the study areas are sexually active before the age of 15 years, and some of them are parents. ${ }^{37}$ Previous research in the study setting has also identified young mothers as a vulnerable group with regards to knowledge on breastfeeding and other infantfeeding practices, hence needy of the intervention. ${ }^{30}$ However, in this study the youngest woman recruited was aged 14 years. (b) Women with disability that would make delivery of the intervention difficult, for example, hearing or sight problem, or intellectual impairment. (c) Women who lost the pregnancy and/or had still-birth. (d) Women who were lost to follow-up during pregnancy. (e) Mother-baby pairs if baby was born with disability that would significantly affect infant feeding, for example, developmental problems. We did not have such cases in actual sense so no children were excluded based on this criteria. Recruitment of the participants was done from September 2012 to February 2014. An eventual sample size of 1110 mother-child pairs was included.

The experimental intervention involved regular visits by CHWs for personalized home-based nutritional counselling of women from the time of recruitment (during pregnancy, continued until the baby attained 1 year). The mothers in the intervention were visited at least once monthly during pregnancy until week 34 after when they were visited every week until giving birth and more often as necessary in the 1 st month after giving birth (for support in initiating breastfeeding and sustaining EBF). They were then visited once every month until the 5th month when they were visited fortnightly (to prepare them for introduction of complementary feeding) and monthly in the subsequent months for 1 year. Counselling encompassed maternal nutrition, immediate initiation of breastfeeding after birth, breast positioning and attachment, EBF, frequency and duration of breastfeeding, expressing breast milk, storage, handling and feeding of expressed breast milk and lactation management. It also included age-appropriate complementary feeding. We did not establish or test the HIV status of participants in this study, but the $\mathrm{CHW}$ in the intervention arm were trained on infant feeding in the context of HIV and were expected to incorporate this in the counselling, without establishing HIV status of the mother. Further, the CHWs were advised to counsel mothers to seek 
further counselling and support at the health facilities in the event they were HIV positive.

CHWs in the CUs, mainly with at least primary level education and basic CHW training by the government, were trained on counselling mothers on appropriate MIYCN practices. In several of the Units, we (through a different APHRC's project) had to facilitate the government to establish CUs, recruit and offer basic training to the CHWs as the CUs were not existent or functional before initiating the intervention. Training of CHWs was done using the Community IYCF Counselling Package developed by UNICEF in partnership with other organizations, which has been adopted by the Kenya Ministry of Health (http://uni.cf/1QavG2g), based on the WHO IYCF integrated course. ${ }^{38}$ The package is designed to equip community workers or primary health care staff to be able to support mothers, fathers and other caregivers to optimally feed their infants and young children. The CHWs were equipped with IYCF counselling cards; brightly coloured illustrations that depict key IYCF concepts and behaviours for the CHWs to share with mothers, fathers and other caregivers. These materials can be obtained in the project's webpage (http://bit.ly/1LBfr7M). CHWs in the control arm were not trained on MIYCN but were trained (through the regular government facilitated training) on standard care, which included antenatal and postnatal care, family planning, delivery with skilled attendance, immunization and community nutrition. Although detailed training on MIYCN including breast positioning and attachment, frequency and duration of breastfeeding, expressing breast milk, storage and handling of expressed breast milk and lactation management, which would help in sustaining EBF was not given to the CHWs in the control group, training on the message of EBF for 6 months and extended breastfeeding for 2 years has increasingly been integrated in the basic CHW training since the inception of the current (2012-2017) MIYCN strategy. In the control arm, the CHWs were expected to visit the mothers according to usual practice which is defined by need, and usually more frequent around the time of birth.

All recruited pregnant women in both the intervention arm and control arm received IEC materials regarding MIYCN through the CHWs, as part of (optimized) standard care. In addition, both the intervention and control groups were provided with standard care counselling by the CHWs. A total of $30 \mathrm{CHWs}$ across the intervention and control arms were involved during the study. The CHWs were given a monthly incentive of KES 3500 ( $\sim$ USD 35), which is within the government's limit of a minimum monthly incentive of KES 2000 (USD 20). Routine supervision was provided to the CHWs by an intervention monitor and the project team.

Data on infant-feeding knowledge, perceptions and practices, anthropometric measurements and morbidity were collected every 2 months during follow-up through interviewer administered questionnaires until the child was 1 year. Data collection was done by a team of 15 experienced field interviewers, who possessed at least secondary school education training, and independent from the CHWs involved in providing MIYCN counselling and support. Details on data collection procedures and other data collected are published. ${ }^{24}$ Data included in the analysis are for children born between December 2012 and July 2014.

\section{Comparison study}

Data for the comparison study comes from the IVP project, which succeeded the Wellcome Trust UPHD-MCH project from 2011 to December 2014. Data included in the comparison study are for children born between September 2012 and February 2014. Apart from continuation of the $\mathrm{MCH}$ longitudinal study, the study aimed to monitor and assess the intended and unintended effects of vaccinations on children in the study area to ensure evidence-based policies for vaccine and preventive drug delivery in LICs, in order to reduce child morbidity and mortality. The study recruited mothers who were residents in the NUDHSS and their children who were born within the study period. The mother-child pair was visited every 4 months between 2011 and 2014 until the youngest child was 8 months. As in the pre-intervention study, efforts were made to recruit the mother-child pairs as early as possible after birth. Information on breastfeeding and other feeding practices, vaccination, health care seeking, health status and anthropometric measurements were also collected.

Both pre-intervention and the comparison studies were run by the same team using similar procedures and questionnaires so that the data quality was similar across all the rounds of data collection. Additional details about the comparison study procedures are published elsewhere. ${ }^{39}$

\section{Research design}

The question we ask and answer here is whether regular $\mathrm{CHW}$ counselling and provision of MIYCN information materials during the Intervention study, regardless of which trial arm, is associated with higher levels of EBF from birth to 6 months compared with the Comparison study. As the Intervention study and comparison study were performed in the same years, a contrast of these two offers some control for secular trends over time in dissemination and health literacy about the importance of EBF. We therefore use the three studies (Pre-intervention, Intervention and Comparison) to construct a quasi-experimental study. We define four study groups: Preintervention group, MIYCN-Intervention group, MIYCNControl group and Comparison group.

\section{Outcome measures}

We defined EBF according to the WHO definition, that is, baby receiving mother's milk only and no other liquids or foods except medicines, minerals and vitamins. ${ }^{40}$ Three outcome measures of interest (EBF at 2, 4 and 6) were constructed. The variable on $\mathrm{EBF}$ was constructed using a series of questions asked longitudinally during the three studies. These included: (i) if the 
child was given anything other than breast milk in the first 3 days of life [i.e., prelacteal feeds (prelacteal feeding refers to feeding on foods other than breast milk in the 1st days after birth before breast milk flow is established)]; (iia) if the child was given anything other than breast milk in the last 3 days before each visit; (iib) at what age the child was started on the food/drink; (iiia) if the child has ever been given anything other than breast milk; (iiib) at what age the child was started on the food/drink. So to determine whether the child was on EBF, we used questions $\mathrm{i}$, iia and iiia. To determine at what age, we used questions $\mathrm{i}$, iib and iiib. For children in the pre-intervention study and the comparison study, depending on when they were first recruited to the study, the mother had to answer the above questions from recall.

\section{Control variables}

Control variables included maternal demographic and socioeconomic status [including age, marital status, education level, ethnicity, religion, parity (number of children ever given birth to; for the Pre-intervention and Comparison studies, this includes the index child; for the intervention study, the index child is not included as the baseline data was collected during pregnancy) and main source of livelihood), and household wealth status, categorized into tertiles. Knowledge of EBF at baseline, used as a proxy for exposure to breastfeeding promotion, was included as a control variable. Other control variables included place of delivery, categorized into two, either at a health facility or otherwise (including home or traditional birth attendant facility) (Table 1).

Data on socio-economic status were either collected at baseline in the respective studies described above or extracted from the NUHDSS database and linked to the study participants through their household identifier, whereas data for the other control variables were collected at baseline from the respective studies described above.

\section{Statistical analysis}

We examined baseline differences among the Pre-intervention group, MIYCN Control group, MIYCN-Intervention group and the Comparison study group using the $\chi^{2}$ test, adjusted for clustering at the $\mathrm{CU}$ (village), being the unit of randomization. The proportion of mother-baby pairs practising EBF was compared among the four groups at 2, 4 and 6 months. The $\chi^{2}$ test was used to check for significant differences in the proportions, adjusting for clustering within a community. We then used logistic regression for the three outcome variables (EBF at 2, 4 and 6 months) to control for baseline characteristics, adjusting for clustering at the $\mathrm{CU}$ (village) using the 'survey' command in Stata. We first fitted bivariate regression models of each of the three outcomes of interest and the predictive variable (indicating whether the child belongs to the Pre-intervention, MIYCN-intervention, MIYCN-Control or Comparison group), and also with each of the control variables listed above (mothers age, education, marital status, ethnicity group, etc.). Using a cut-off of $P$-value $=0.25$, we selected the variables to be included in the final multiple logistic regressions. We did not control for multiple births, nor did we exclude pre-term or low-birth weight children. Children of the same mother in the different studies, for example, pre-intervention and intervention were included in the analysis, and the baseline data collected with respect to each child were used in the analysis.

\section{Results}

The study involves 5824 mother-child pairs in the Pre-intervention study; 1110 mother-child pairs in the Intervention Study, 521 in the intervention arm and 581 in the control arm; and 487 mother-child pairs in the Comparison study. Children in the Pre-intervention study were born between September 2006 and December 2011; and the Intervention and parallel studies between September 2012 and July 2014.

\section{Baseline characteristics}

The baseline distribution of participants by demographic and socio-economic variables between the four groups is presented in Table 1. The distributions showed significant difference in basic socio-demographic factors among the four groups for some variables including maternal age, maternal education level, main source of income, socio-economic status, knowledge on duration of EBF at baseline and place of delivery $(P<0.05)$, respectively.

\section{$E B F$}

Table 2 shows the proportions of children that were exclusively breastfed for 2, 4 and 6 months, measured cumulatively.

There was higher prevalence of reported EBF at all the study points (2, 4 and 6 months) among children in the MIYCNIntervention and MIYCN-Control groups compared with the Pre-intervention group and the Comparison group. The prevalence of EBF was also slightly higher in the Comparison group compared with the Pre-intervention group at all the study points. At 6 months, the prevalence of EBF was $2 \%$ in the Pre-intervention group compared with $55 \%$ in the MIYCN-Intervention group, $55 \%$ in the MIYCN-Control group and $3 \%$ in the Comparison group.

\section{Regression analysis for $E B F$}

The odds ratio (OR) of EBF was higher in all the groups (MIYCN-Intervention, MIYCN-Control and the Comparison group compared with the Pre-intervention group) at 2, 4 and 6 months.

At 6 months, the unadjusted OR was 64.4 (95\% CI 46.7-88.7), 66.1 (95\% CI 32.3-135.4) and 1.7 (95\% CI 0.9-3.2) for the MIYCN-Control, MIYCN-Intervention and Comparison group, respectively, compared with the Preintervention group. After adjusting for baseline characteristics, 
Table 1. Baseline distribution of the study participants by demographic and socio-economic variables by study group, MIYCN (Maternal, Infant and Young Child Nutrition)-quasi-experimental study, Nairobi slums, 2015

\begin{tabular}{|c|c|c|c|c|c|c|c|c|c|}
\hline & \multirow{2}{*}{\multicolumn{2}{|c|}{$\begin{array}{c}\text { Pre-intervention (2007-2011) } \\
\text { Total } \\
\end{array}$}} & \multicolumn{4}{|c|}{ Intervention (MIYCN 2012-2015) } & \multirow{2}{*}{\multicolumn{2}{|c|}{$\begin{array}{c}\text { Comparison (2012-2014) } \\
\text { Total } \\
\end{array}$}} & \multirow[b]{3}{*}{$P$-values $^{\mathrm{a}}$} \\
\hline & & & \multicolumn{2}{|c|}{ Control } & \multicolumn{2}{|c|}{ Intervention } & & & \\
\hline & $n$ & $\%$ & $n$ & $\%$ & $n$ & $\%$ & $n$ & $\%$ & \\
\hline \multicolumn{10}{|l|}{ Child's sex } \\
\hline Male & 2948 & 50.6 & 302 & 52.8 & 262 & 50.2 & 267 & 54.8 & \\
\hline Female & 2876 & 49.4 & 270 & 47.2 & 260 & 49.8 & 220 & 45.2 & 0.172 \\
\hline Missing & 0 & & 9 & & 7 & & 0 & & \\
\hline \multicolumn{10}{|l|}{ Mother's age (years) } \\
\hline $14-20$ & 1365 & 25.0 & 158 & 27.5 & 159 & 30.5 & 66 & 16.6 & \\
\hline $21-24$ & 1656 & 30.3 & 177 & 30.8 & 164 & 31.5 & 101 & 25.4 & \\
\hline $25-29$ & 1420 & 26.0 & 137 & 23.9 & 133 & 25.5 & 124 & 31.2 & \\
\hline $30-45$ & 1028 & 18.8 & 102 & 17.8 & 65 & 12.5 & 107 & 26.9 & 0.000 \\
\hline Missing & 355 & & 7 & & 8 & & 89 & & \\
\hline \multicolumn{10}{|l|}{ Marital status } \\
\hline Married & 901 & 15.5 & 70 & 12.2 & 75 & 14.5 & 60 & 12.7 & \\
\hline Not married & 4914 & 84.5 & 504 & 87.8 & 444 & 85.5 & 414 & 87.3 & 0.265 \\
\hline Missing & 9 & & 7 & & 10 & & 13 & & \\
\hline \multicolumn{10}{|c|}{ Highest level of education completed } \\
\hline Less than primary & 182 & 3.4 & 112 & 19.7 & 85 & 16.8 & 15 & 3.9 & \\
\hline Primary school & 3875 & 72.4 & 327 & 57.6 & 284 & 56.2 & 242 & 63.4 & \\
\hline Secondary school & 1297 & 24.2 & 129 & 22.7 & 136 & 26.9 & 125 & 32.7 & 0.000 \\
\hline Missing & 470 & & 13 & & 24 & & 105 & & \\
\hline \multicolumn{10}{|c|}{ Main source of livelihood } \\
\hline Business & 626 & 12.2 & 65 & 11.5 & 66 & 13.1 & 32 & 6.6 & \\
\hline Informal & 377 & 7.4 & 60 & 10.6 & 31 & 6.1 & 16 & 3.3 & \\
\hline Formal & 64 & 1.3 & 24 & 4.2 & 55 & 10.9 & 4 & 0.8 & \\
\hline Unemployed & 4051 & 79.2 & 418 & 73.7 & 353 & 69.9 & 435 & 89.3 & 0.000 \\
\hline Missing & 706 & & 14 & & 24 & & 0 & & \\
\hline \multicolumn{10}{|c|}{ Ethnicity of the mother } \\
\hline Kikuyu & 1401 & 25.6 & 119 & 26.2 & 132 & 30.2 & 85 & 21.4 & \\
\hline Luhya & 962 & 17.6 & 92 & 20.2 & 73 & 16.7 & 74 & 18.6 & \\
\hline Luo & 1046 & 19.1 & 66 & 14.5 & 81 & 18.5 & 64 & 16.1 & \\
\hline Kamba & 1136 & 20.8 & 96 & 21.1 & 80 & 18.3 & 95 & 23.9 & \\
\hline Other & 926 & 16.9 & 82 & 18.0 & 71 & 16.2 & 80 & 20.1 & 0.866 \\
\hline Missing & 353 & & 126 & & 92 & & 89 & & \\
\hline \multicolumn{10}{|c|}{ Total number of children ever given birth } \\
\hline Null & 1820 & 31.3 & 212 & 36.5 & 223 & 42.2 & 131 & 26.9 & \\
\hline One & 1682 & 28.9 & 178 & 30.6 & 165 & 31.2 & 159 & 32.6 & \\
\hline Two+ & 2311 & 39.8 & 191 & 32.9 & 141 & 26.7 & 197 & 40.5 & 0.000 \\
\hline Missing & 11 & & 0 & & 0 & & 0 & & \\
\hline \multicolumn{10}{|l|}{ Wealth tertiles } \\
\hline Lowest & 1655 & 30.6 & 147 & 35.8 & 122 & 30.0 & 115 & 29.0 & \\
\hline Middle & 1816 & 33.6 & 139 & 33.8 & 141 & 34.7 & 147 & 37.1 & \\
\hline Highest & 1940 & 35.9 & 125 & 30.4 & 143 & 35.2 & 134 & 33.8 & 0.760 \\
\hline Missing & 413 & & 170 & & 123 & & 91 & & \\
\hline
\end{tabular}




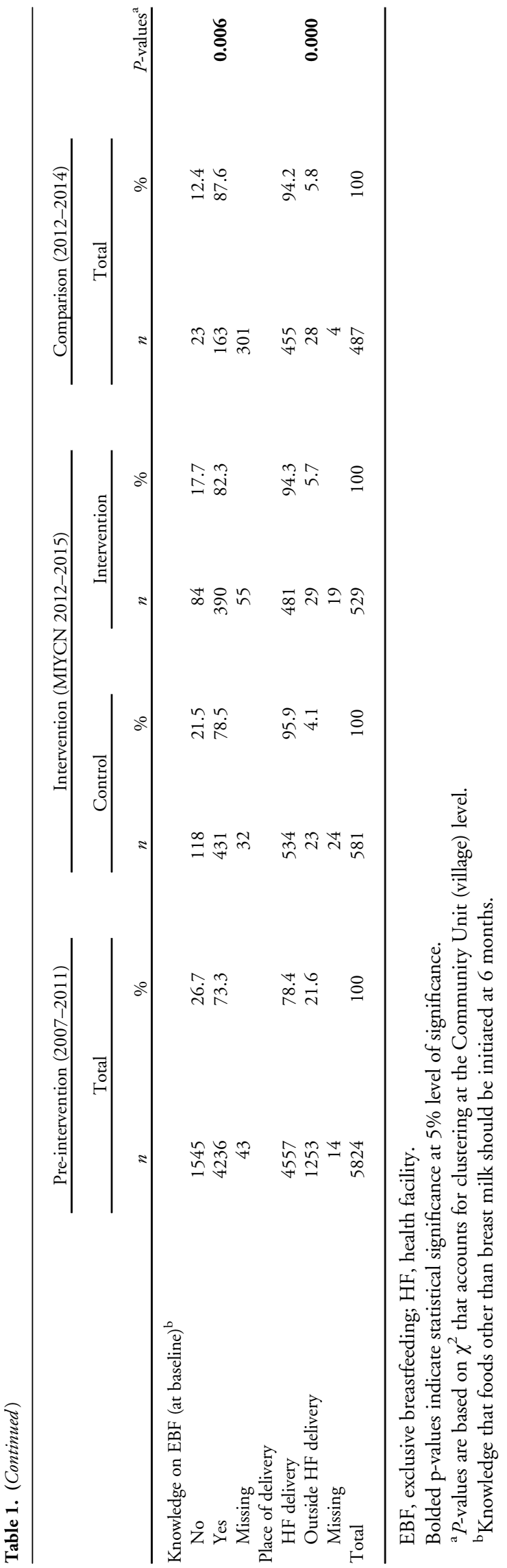

the OR for EBF at 6 months was 66.9 (95\% CI 45.4-96.4), 84.3 (95\% CI 40.7-174.6) and 3.9 (95\% CI 1.8-8.4), respectively.

At 4 months, the unadjusted OR was 15.5 (95\% CI 12.2-19.7), 16.0 (95\% CI 7.9-32.4) and 1.3 (95\% CI 1.0-1.7) for the MIYCN-Control, MIYCN-Intervention and Comparison group, respectively, compared with the Preintervention group. After adjusting for baseline characteristics, the OR for EBF at 4 months was 16.1 (95\% CI 11.6-22.3), 21.1 (95\% CI 11.6-38.5) and 3.5 (95\% CI 2.5-4.8), respectively.

At 2 months, the unadjusted OR was 13.3 (95\% CI 9.4-18.7), 17.1 (95\% CI 9.1-32.2) and 1.1 (95\% CI 0.8-1.5) for the MIYCN-Control, MIYCN-Intervention and Comparison group, respectively, compared with the Pre-intervention group. After adjusting for baseline characteristics, the OR for $\mathrm{EBF}$ at 2 months was 13.7 (95\% CI 8.4-22.4), 22.6 (95\% CI 11.21-45.4) and 4.3 (95\% CI 3.1-5.9), respectively (Table 3).

\section{Discussion}

This paper presents results of a quasi-experimental study designed to control for secular trends in EBF, and constructed using data from a Pre-intervention study done in 2007-2011, an Intervention study in 2012-2014 and a Comparison study in 2012-2015. We aimed to determine potential effectiveness of the Kenya national Community Health Strategy to promote EBF from birth to 6 months in urban poor settings in Nairobi, Kenya, through home-based visits by CHWs. We found that the intervention, involving CHW visits about infant health with general and specific counselling about EBF had a high impact on EBF for the first 6 months. The effect was seen among mothers in both the control and intervention arms of the Intervention study, but not in the group in the Comparison study, done at the same time, where CHWs were not financially motivated to visit mothers, or did not exist. This may indicate that the difference observed in EBF was due to incentivizing and routinely supervising the $\mathrm{CHWs}$, hence motivating them to regularly visit and counsel the mothers. In effect, this may mean that optimizing standard care, through incentivizing and supervising CHWs within the government Community Health Strategy to conduct home-based counselling of mothers would be effective in promoting EBF, particularly in underserved areas such as urban poor settings.

The study shows that there was remarkable improvement in EBF rates for children in both the intervention arm and the control arm of the MIYCN intervention study, from $2 \%$ at pre-intervention to over $50 \%$ in both arms following the intervention. However, this study shows a small (though significant) difference between the Pre-intervention group and the Comparison group from $2 \%$ at pre-intervention to $3 \%$ during the intervention period. The situation of EBF in Kenya has improved at the national level, from $32 \%$ in 2008 to $61 \%$ in 2014, according to the latest Kenya Demographic and Health Survey (KDHS). ${ }^{41}$ This may be a result of the changes 
Table 2. Practice of exclusive breastfeeding (EBF) by study group, Maternal, Infant and Young Child Nutrition-quasi-experimental study, Nairobi slums, 2015

\begin{tabular}{|c|c|c|c|c|c|c|c|c|c|}
\hline & \multirow{2}{*}{\multicolumn{2}{|c|}{ Pre-intervetion (2007-2011) }} & \multicolumn{4}{|c|}{ Intervention (2012-2015) } & & & \multirow[b]{3}{*}{$P$-values ${ }^{\mathrm{a}}$} \\
\hline & & & \multicolumn{2}{|c|}{ Control } & \multicolumn{2}{|c|}{ Intervention } & \multicolumn{2}{|c|}{ Comparison (2012-2014) } & \\
\hline & $n$ & $\%$ & $n$ & $\%$ & $n$ & $\%$ & $n$ & $\%$ & \\
\hline \multicolumn{10}{|c|}{ EBF for the $0-2$ months } \\
\hline No & 4457 & 77.2 & 107 & 20.3 & 78 & 16.5 & 369 & 75.8 & \\
\hline Yes & 1314 & 22.8 & 419 & 79.7 & 394 & 83.5 & 118 & 24.2 & 0.000 \\
\hline \multicolumn{10}{|c|}{ EBF for the $0-4$ months } \\
\hline No & 5007 & 87.2 & 149 & 30.6 & 131 & 29.9 & 410 & 84.2 & \\
\hline Yes & 734 & 12.8 & 338 & 69.4 & 307 & 70.1 & 77 & 15.8 & 0.000 \\
\hline \multicolumn{10}{|c|}{ EBF for $0-6$ months } \\
\hline No & 5624 & 98.2 & 208 & 45.4 & 188 & 44.8 & 472 & 96.9 & \\
\hline Yes & 105 & 1.8 & 250 & 54.6 & 232 & 55.2 & 15 & 3.1 & 0.000 \\
\hline Total & 5824 & 100 & 581 & 100 & 529 & 100 & 487 & 100 & \\
\hline
\end{tabular}

${ }^{\text {a }} P$-values are computed after excluding the missing/do not knows.

in policy and practice. The message of EBF for 6 months and extended breastfeeding for 2 years has increasingly been integrated in the basic CHW training since the inception of the current (2012-2017) MIYCN strategy. In addition, an Act of Parliament (Breast Milk Substitutes - BMS Act 2012 - http:// bit.ly/1FXsEua) that regulates and controls marketing and distribution of breast milk substitutes in Kenya in order to protect breastfeeding was enacted in 2012. Another key policy change was the introduction of the free maternity policy (http://bit.ly/1QsLuZ2) since June 2013 with the placement of the new government, under which maternity care is now provided for free in public health facilities nationally. The free maternity policy is likely to promote breastfeeding through counselling of mothers delivering at the health facilities by health care workers. However, urban poor settings remain unreached due to poor access to public health care (given limited public health facilities) and other social and economic factors. ${ }^{30}$ For example, the community strategy was hardly functional in the slums where we conducted the study before this project, and we had to assist the government to set-up CUs to enable the intervention study. The enactment of the BMS Act may not have a large impact on the urban poor particularly because there is limited use of commercially produced substitutes such as infant formula due to the prohibitive cost; according to results from qualitative formative research that we carried out before the intervention. The free maternity health policy may not have had a big impact in urban poor settings due to the unavailability of public health facilities in the urban slums (where free maternity services are offered and initiatives such as the BFHI are implemented). Health facilities in urban poor settings are generally (substandard) privately owned. ${ }^{42}$ A formative qualitative study done before the intervention indicated that there are complex socio-economic factors in urban poor settings that make it difficult for women to adhere to the WHO recommendations for breastfeeding including women having to resume paid work shortly after delivery. ${ }^{30}$ Although there may be ways of ensuring working women breastfeed such as encouraging them to express breast milk to be fed to the baby in their absence, the formative study found that expressing breast milk is a rare practice in these settings due to cultural unacceptability and other factors such as fear of contamination due to lack of appropriate storage facilities such as refrigerators. ${ }^{30}$ Although we incorporated counselling on expressing breast milk in the intervention, few women reported doing it. Another potential reason for low prevalence of EBF in the Comparison group compared with the prevalence at the country level may also be attributable to the fact that the method in our study is more rigorous in establishing exclusivity of breastfeeding than the 24-hour recall method used in the KDHS. In addition, we report cumulative EBF from birth to 6 months, following the children longitudinally, whereas the KDHS reports EBF for children aged 0-6 months (crosssectionally). Therefore, our results on EBF for 6 months may not be directly comparable with those reported in the KDHS.

The large difference between the MIYCN intervention groups and the comparison group may be attributed to regular CHWs' visits for counselling and support and distribution of information materials to the mothers in both intervention and control areas, motivated by incentivizing CHWs to visit mothers in the study setting, and supervising them, hence optimizing the proposed standard primary health care that is hampered by lack of CHW motivation. This is supported by other evidence in LMICs that indicate that scaling up and sustaining CHW programmes in LMICs requires adequate training, motivation and supervision among other things. ${ }^{43}$ Findings from our qualitative midline and endline studies within the MIYCN Intervention study indicate that women in both control and intervention groups were very impressed with 
Table 3. Logistic regression for exclusive breastfeeding (EBF) for 6 months by study group, controlling for baseline characteristics, Maternal, Infant and Young Child Nutrition-quasi-experimental study, Nairobi slums, 2015

\begin{tabular}{|c|c|c|c|c|c|c|c|c|c|}
\hline & \multicolumn{3}{|c|}{6 months } & \multicolumn{3}{|c|}{4 months } & \multicolumn{3}{|c|}{2 months } \\
\hline & $\mathrm{OR}^{\mathrm{a}}$ & $P$-values & $95 \% \mathrm{CI}$ & $\mathrm{OR}^{\mathrm{a}}$ & $P$-values & $95 \% \mathrm{CI}$ & $\mathrm{OR}^{\mathrm{a}}$ & $P$-values & $95 \% \mathrm{CI}$ \\
\hline \multicolumn{10}{|c|}{ Group (ref: Pre-intervention 2007-2011) } \\
\hline Control (2012-2015) & 66.94 & 0.000 & {$[45.01,99.54]$} & 16.1 & 0.000 & {$[11.62,22.29]$} & 13.71 & 0.000 & {$[08.41,22.35]$} \\
\hline Intervention (2012-2015) & 84.26 & 0.000 & {$[40.65,174.63]$} & 21.13 & 0.000 & {$[11.61,38.45]$} & 22.56 & 0.000 & {$[11.21,45.43]$} \\
\hline Parallel (2012-2014) & 3.91 & 0.002 & {$[01.81,08.42]$} & 3.47 & 0.000 & {$[02.50,04.81]$} & 4.27 & 0.000 & {$[03.07,05.93]$} \\
\hline \multicolumn{10}{|l|}{ Child's sex (ref: male) } \\
\hline Female & 1.02 & 0.907 & {$[00.76,01.36]$} & 0.95 & 0.419 & {$[00.82,01.09]$} & 0.94 & 0.521 & {$[00.76,01.16]$} \\
\hline \multicolumn{10}{|c|}{ Mother's age in years (ref: $14-20$ years) } \\
\hline $21-24$ & 0.77 & 0.110 & {$[00.55,01.07]$} & 0.99 & 0.968 & {$[00.71,01.39]$} & 0.93 & 0.600 & {$[00.69,01.25]$} \\
\hline $25-29$ & 0.75 & 0.078 & {$[00.54,01.04]$} & 1.1 & 0.541 & {$[00.78,01.55]$} & 1.04 & 0.780 & {$[00.79,01.36]$} \\
\hline $30-45$ & 0.84 & 0.495 & {$[00.49,01.44]$} & 1.24 & 0.178 & {$[00.89,01.72]$} & 1.1 & 0.532 & {$[00.80,01.51]$} \\
\hline \multicolumn{10}{|c|}{ Marital status (ref not married/in union) } \\
\hline Married/in union & 1.22 & 0.278 & {$[00.84,01.77]$} & 0.95 & 0.619 & {$[00.78,01.16]$} & 1.12 & 0.310 & {$[00.89,01.42]$} \\
\hline \multicolumn{10}{|c|}{ Highest level of education completed (ref: < primary) } \\
\hline Primary school & 1.23 & 0.178 & {$[00.90,01.68]$} & 1.25 & 0.244 & {$[00.84,01.84]$} & 1.66 & 0.006 & {$[01.19,02.32]$} \\
\hline Secondary school & 1.31 & 0.261 & {$[00.80,02.16]$} & 1.36 & 0.097 & {$[00.94,01.98]$} & 1.89 & 0.001 & {$[01.39,02.56]$} \\
\hline \multicolumn{10}{|c|}{ Main source of livelihood (ref: business) } \\
\hline Informal & 1 & 0.985 & {$[00.57,01.77]$} & 0.85 & 0.520 & {$[00.50,01.44]$} & 0.79 & 0.261 & {$[00.52,01.21]$} \\
\hline Formal & 0.39 & 0.020 & {$[00.18,00.84]$} & 0.46 & 0.075 & {$[00.19,01.09]$} & 1.17 & 0.582 & {$[00.65,02.10]$} \\
\hline Unemployed & 1.36 & 0.071 & {$[00.97,01.91]$} & 1.52 & 0.003 & {$[01.19,01.94]$} & 1.43 & 0.006 & {$[01.13,01.81]$} \\
\hline \multicolumn{10}{|c|}{ Ethnicity of the mother (ref: Kikuyu) } \\
\hline Luhya & 0.78 & 0.311 & {$[00.47,01.30]$} & 0.77 & 0.011 & {$[00.63,00.93]$} & 0.74 & 0.013 & {$[00.60,00.93]$} \\
\hline Luo & 0.66 & 0.038 & {$[00.45,00.97]$} & 0.66 & 0.003 & {$[00.52,00.84]$} & 0.67 & 0.000 & {$[00.57,00.79]$} \\
\hline Kamba & 0.94 & 0.761 & {$[00.60,01.46]$} & 0.93 & 0.452 & {$[00.75,01.14]$} & 1.07 & 0.508 & {$[00.87,01.30]$} \\
\hline Other & 0.68 & 0.094 & {$[00.43,01.08]$} & 0.97 & 0.795 & {$[00.76,01.24]$} & 1.02 & 0.830 & {$[00.81,01.29]$} \\
\hline \multicolumn{10}{|l|}{ Parity (ref: no child) } \\
\hline One & 1.25 & 0.289 & {$[00.81,01.91]$} & 1.4 & 0.002 & {$[01.16,01.69]$} & 1.46 & 0.007 & {$[01.13,01.87]$} \\
\hline Two+ & 1.75 & 0.110 & {$[00.86,03.53]$} & 1.43 & 0.015 & {$[01.09,01.87]$} & 1.53 & 0.000 & {$[01.30,01.80]$} \\
\hline \multicolumn{10}{|l|}{ Wealth tertile (ref: lowest) } \\
\hline Middle & 0.76 & 0.387 & {$[00.40,01.46]$} & 0.83 & 0.230 & {$[00.60,01.14]$} & 0.89 & 0.179 & {$[00.74,01.06]$} \\
\hline Upper & 0.77 & 0.349 & {$[00.43,01.38]$} & 0.71 & 0.018 & {$[00.54,00.93]$} & 0.84 & 0.126 & {$[00.67,01.06]$} \\
\hline \multicolumn{10}{|c|}{ Knowledge on EBF at baseline (ref: no) } \\
\hline Yes & 1.36 & 0.173 & {$[00.86,02.14]$} & 2.79 & 0.000 & {$[02.02,03.84]$} & 2.38 & 0.000 & {$[01.73,03.27]$} \\
\hline \multicolumn{10}{|c|}{ Place of delivery (ref: health facility) } \\
\hline Outside health facility & 0.63 & 0.112 & {$[00.35,01.13]$} & 0.5 & 0.000 & {$[00.37,00.69]$} & 0.52 & 0.000 & {$[00.41,00.65]$} \\
\hline & 5315 & & & 5346 & & & 5399 & & \\
\hline
\end{tabular}

${ }^{\mathrm{a}}$ Odds ratio $(\mathrm{OR})$ adjusted for baseline characteristics and clustering. 
the $\mathrm{CHW}$ visits, including frequency of visits. They indicated that $\mathrm{CHW}$ save them useful advice, which they often followed or which informed important decisions such as regarding resuming paid work, and some women had to make important decision, such as delaying resuming work. Women not in the intervention study were unlikely to receive $\mathrm{CHW}$ visits and information materials at the community level as a key challenge in the actualization of the Community Health Strategy in Kenya is lack of incentives for CHWs. ${ }^{23}$ The monthly financial incentive given to the CHWs in our study was within the range proposed in the government Community Health Strategy, and was agreed upon in consultation with the government officials.

The findings of this study give an indication of potential effectiveness of the government's Community Health Strategy in promoting optimal breastfeeding practices, and in particular EBF in the urban poor settings. This is particularly important given the change in the government's MIYCN strategy to adopt the BFCI, which proposes use of CHWs to promote optimal MIYCN practices at the community level, to complement the BFHI, which promotes optimal MIYCN at the health facility level. The study findings may indicate that optimized standard care involving home-based visits by incentivized CHWs with basic training, supervision and provision of MIYCN information materials could be adequate to change breastfeeding practices This is because there was little difference in the EBF changes over 6 months between the two arms of the MIYCN intervention. Although we did not train CHWs in the control arm on MIYCN, an endline evaluation of knowledge levels on MIYCN among CHWs (unpublished) indicated high and similar levels of knowledge across the two groups. It is possible that the CHWs in the control group may have obtained this knowledge on MIYCN form the basic training (as it also includes messages on EBF) or from other sources, for example, other NGOs. Our anticipation was that the more detailed training on MIYCN including breast positioning and attachment, frequency and duration of breastfeeding, expressing breast milk, storage and handling of expressed breast milk and lactation management, which would help in sustaining EBF would make a difference. The results do not seem to indicate that this extra training made a difference. Further, we also anticipate that there could have been contamination due to the information materials that we provided to the CHWs in both arms, which may have enhanced knowledge of CHWs in both intervention and control groups. Contamination between the two groups could also have happened through possible interaction of the CHWs across the arms and possible sharing of the counselling materials that we provided to the intervention group, given that it was not possible to blind the CHWs regarding the purpose of the intervention. This is particularly possible because, in trying to be pragmatic, we used existing CHWs within the government's Community Health Strategy, who live in the same community in close proximity with each other, and who, being within the same government programme may have met regularly. Further investigation through analysis of qualitative data collected at midline and endline from
CHWs and mothers in both arms of the Intervention study is likely to offer some additional information on the pathways through which the intervention worked in both arms.

The level of increase in EBF documented in this study was also documented in a study in Bangladesh, where home-based breastfeeding counselling using peer counsellors was highly effective in improving breastfeeding practices, with more than 10-fold increase in EBF rate for 6 months in the intervention group compared with the control group. ${ }^{44}$ Similarly, the study agrees with findings of a pre-post study without control conducted in an agrarian rural setting in Kenya by Wangalwa et al., ${ }^{45}$ which found effectiveness of the Community Strategy (using CHWs for home-based care) in improving $\mathrm{MCH}$ outcomes, including EBF for the first 6 months from 20\% at baseline to $52 \%$ post-intervention. Other studies elsewhere, such as in Ghana and Brazil, have also documented the importance of home-based counselling on the rate of EBF. ${ }^{46-50}$ For example, in Ghana, $40 \%$ prevalence of EBF was reported among women receiving perinatal and postnatal (in the first 6 months after birth) counselling compared with control group (20\%) not receiving the counselling. ${ }^{46}$ In Brazil, Courtinho et $a l^{47}$ found that a huge effect in sustained breastfeeding in the group combined BFHI plus home-based visits for up to 6 months after discharge. The study found that the BFHI achieved a high rate of $70 \%$ in EBF in the hospital compared with $21 \%$ before intervention but this was not sustained at home in the group without community-based support. The patterns of EBF in the two groups for 10-180 days differed significantly with a mean aggregate prevalence of $13 \%$ in the BFHI alone group compared with $45 \%$ in the BHI plus home visits group. ${ }^{47}$ Bhutta et al. ${ }^{1}$ identified community-based interventions using $\mathrm{CHWs}$ as an important delivery model for nutrition education and promotion, particularly among difficult to reach populations.

Strengths of this study include being able to constitute a quasi-experimental study using longitudinal studies (pre- and post-intervention), which used similar data collection tools/ questions regarding EBF, to determine effectiveness of an intervention. We also observe EBF from birth to 6 months cumulatively. Limitations in this study may include potential bias in reporting of the primary outcome (EBF), often associated with self-reported outcomes, particularly due to social desirability. Social desirability was plausible as the outcome measure of interest was self-reported, and it was hard to control for this bias, given that mothers in both intervention and control arm were visited by CHWs. However, the fact that we asked several questions longitudinally to determine whether the child was exclusively breastfeeding may partly counter this bias. In addition, the data collection team was different from the CHWs that provided the counselling, which helps to reduce the social desirability bias. Another limitation may be in the differences in the design of the three studies to constitute the quasi-experimental study. Though similar questions were asked to the mothers to establish EBF, the intervention groups were recruited during pregnancy and followed up more regularly, 
whereas mothers in the Pre-intervention and the Comparison study groups were recruited after birth and had fewer follow-up visits, meaning there would be longer recall periods to remember when EBF ceased.

In summary, the results indicate potential effectiveness of the Government of Kenya's Community Health Strategy, through CHWs to improve EBF in urban poor settings. The results of this study will inform policy and practice particularly regarding implementation of the proposed national BFCI programme, which proposes to promote MIYCN through the Community Health Strategy to complement promotion of MIYCN through the BFHI at the health facility level. Although this study offers a great contribution to implementation science, more investigation into pathways through which the intervention worked is worthwhile in order to inform the implementation of the BFCI programme in Kenya and beyond more appropriately. Increase in EBF for infants living in urban poor settings may improve their health, growth and cognitive development, and their future health and economic productivity. This in effect would not only benefit the child but the whole community through intergenerational transfer of the benefits. This community approach is applicable in other similar African settings.

\section{Acknowledgements}

The authors would like to thank Dr Eliya Zulu, Dr Jean Christophe Fotso, Prof. John Cleland, Prof. Jane Falkingham and Prof. Philippe Bocquier for their contribution to the design of the pre-intervention study. The authors thank Dr George Mgomella, Dr Hilda Essendi and Teresa Saliku for coordination of the pre-intervention study. The authors are grateful to the Unit of Nutrition and Dietetics and the Unit of Community Health Services of the Ministry of Health, Kenya, for their guidance in the design of the intervention project and their continued support of the implementation of the intervention project including in identification and training of CHWs and provision of educational materials for the intervention. The authors would also like to thank UNICEF, Concern World Wide, the Urban Nutrition Working Group and the Nutrition Information Working Group, among other agencies/NGOs/groups for their guidance in the design of the intervention study. The authors are highly indebted to the data collection and management team and the study participants.

\section{Financial Support}

This study was funded by the Wellcome Trust, Grant No. 078530/Z/05/Z (Pre-intervention study) and Grant No. 097146/Z/11/Z (Intervention Study) and DANIDA, Grant No. IND0912010 (Comparison study). This research was also made possible through the generous funding for the NUHDSS by the Bill and Melinda Gates Foundation (Grant No. OPP1021893) and core funding for APHRC by The William and Flora Hewlett Foundation (Grant No. 2009-40510), and the Swedish International Cooperation
Agency (Grant No. 2011-001578). P.L.G. was supported by a British Academy mid-career fellowship (Ref: MD120048).

\section{Conflicts of Interest}

None.

\section{Ethical Standards}

The authors assert that all procedures contributing to this work comply with the ethical standards set by the Kenya Medical Research Institute Ethical Review Committee on research regarding human subjects and with the Helsinki Declaration. The investigators upheld the fundamental principles regarding research on human subjects: respect for persons, beneficence and justice. For all data collection activities, written informed consent was obtained from the eligible participants following full disclosure regarding the study before data collection was done.

\section{References}

1. Bhutta ZA, Das JK, Rizvi A, et al. Evidence-based interventions for improvement of maternal and child nutrition: what can be done and at what cost? Lancet. 2013; 382, 452-477.

2. Bhutta ZA, Ahmed T, Black RE, et al. What works? Interventions for maternal and child undernutrition and survival. Lancet. 2008; 371, 417-440.

3. Gareth J, Richard WS, Robert EB, Zulfiqar AB, Saul SM. How many child deaths can we prevent this year? Lancet. 2003; 362, 65-71.

4. Kramer MS, Kakuma R. The optimal duration of exclusive breastfeeding: a systematic review. Adv Exp Med Biol. 2004; 554, 63-77.

5. Oddy WH, Kendall GE, Blair E, et al. Breast feeding and cognitive development in childhood: a prospective birth cohort study. Paediatr Perinat Epidemiol. 2003; 17, 81-90.

6. WHO. The optimal duration of exclusive breastfeeding. Report of an Expert Consultation, 2002. WHO, Geneva.

7. Arifeen S, Black RE, Antelman G, Baqui A, Caulfield L, Becker S. Exclusive breastfeeding reduces acute respiratory infection and diarrhea deaths among infants in Dhaka slums. Pediatrics 2001; 108, E67.

8. WHO. Global Strategy for Infant and Young Child Feeding. 2003. WHO: Geneva.

9. Diallo FB, Bell L, Moutquin JM, Garant MP. The effects of exclusive versus non-exclusive breastfeeding on specific infant morbidities in Conakry. Pan Afr Med J. 2009; 2, 2.

10. Bahl R, Frost C, Kirkwood BR, et al. Infant feeding patterns and risks of death and hospitalization in the first half of infancy: multicentre cohort study. Bull World Health Organ. 2005; 83, 418-426.

11. Victora CG, Horta BL, de Mola CL, et al. Association between breastfeeding and intelligence, educational attainment, and income at 30 years of age: a prospective birth cohort study from Brazil. Lancet Glob Health. 2015; 3, e199-e205.

12. Martens PJ. What do Kramer's Baby-Friendly Hospital Initiative PROBIT studies tell us? A review of a decade of research. J Hum Lact. 2012; 28, 335-342. 
13. Perez-Escamilla R. Evidence based breast-feeding promotion: the Baby-Friendly Hospital Initiative. J Nutr. 2007; 137, 484-487.

14. Merten S, Dratva J, Ackermann-Liebrich U. Do baby-friendly hospitals influence breastfeeding duration on a national level? Pediatrics. 2005; 116, e702-e708.

15. Braun ML, Giugliani ER, Soares ME, et al. Evaluation of the impact of the baby-friendly hospital initiative on rates of breastfeeding. Am J Public Health. 2003; 93, 1277-1279.

16. Montagu D, Yamey G, Visconti A, Harding A, Yoong J. Where do poor women in developing countries give birth? A multi-country analysis of demographic and health survey data. PLoS One. 2011; 6, e17155.

17. Kenya National Bureau of Statistics and ICF Macro. Kenya demographic and health survey 2008-09, 2009. KNBS and ICF Macro, Calverton, MD.

18. CBS Kenya, Ministry of Health [Kenya], ORC Macro. Kenya demographic and health survey 2003: key findings, 2004. CBS, $\mathrm{MOH}$ and ORC Macro, Calverton, MD.

19. Kimani-Murage E, Madise N, Fotso J-C, et al. Patterns and determinants of breastfeeding and complementary feeding practices in urban informal settlements, Nairobi Kenya. BMC Public Health. 2011; 11, 396.

20. Ministry of Public Health and Sanitation. National Strategy on Infant and Young Child Feeding Strategy 2007-2010. 2007. Ministry of Public Health and Sanitation: Nairobi, Kenya.

21. Jelliffe DB, Jelliffe EFP. Programmes to Promote Breastfeeding, 1st edn, 1988. Oxford University Press: Oxford and New York, NY.

22. Ministry of Health [Kenya]. Taking the Kenya essential package for health to the community: a strategy for the delivery of level one services, 2006. Ministry of Health, Kenya.

23. United Nations Children's Fund. Evaluation Report of the Community Health Strategy Implementation in Kenya. 2010. UNICEF: Nairobi.

24. Kimani-Murage EW, Kyobutungi C, Ezeh AC, et al. Effectiveness of personalised, home-based nutritional counselling on infant feeding practices, morbidity and nutritional outcomes among infants in Nairobi slums: study protocol for a cluster randomised controlled trial. Trials. 2013; 14, 445.

25. African Population and Health Research Center. Population and health dynamics in Nairobi's informal settlements, 2002. APHRC, Nairobi.

26. African Population and Health Research Center. Population and health dynamics in Nairobi's informal settlements: report of the Nairobi crosssectional slums survey (NCSS) 2012, 2014. APHRC, Nairobi.

27. Kimani-Murage EW, Fotso JC, Egondi T, et al. Trends in childhood mortality in Kenya: the urban advantage has seemingly been wiped out. Health Place. 2014; 29C, 95-103.

28. Mberu BU, Ciera JM, Elungata P, Ezeh AC. Patterns and determinants of poverty transitions among poor urban households in Nairobi. Afr Dev Rev. 2014; 26, 172-185.

29. Mutua MK, Kimani-Murage E, Ettarh RR. Childhood vaccination in informal urban settlements in Nairobi, Kenya: who gets vaccinated? BMC Public Health. 2011; 11, 6.

30. Kimani-Murage EW, Wekesah F, Wanjohi M, et al. Factors affecting actualisation of the WHO breastfeeding recommendations in urban poor settings in Kenya. Matern \& Child Nutr. 2015; 11, 314-332.

31. Kimani-Murage EW, Schofield L, Wekesah F, et al. Vulnerability to food insecurity in urban slums: experiences from Nairobi, Kenya. J Urban Health. 2014; 91, 1098-1113.
32. Kimani-Murage EW, Muthuri SK, Oti SO, Mutua MK, van de Vijver S, Kyobutungi C. Evidence of a double burden of malnutrition in urban poor settings in Nairobi, Kenya. PLoS One. 2015; 10, e0129943.

33. Fotso JC, Madise N, Baschieri A, et al. Child growth in urban deprived settings: does household poverty status matter? At which stage of child development? Health Place. 2012; 18, 375-384.

34. Emina J, Beguy D, Zulu EM, et al. Monitoring of health and demographic outcomes in poor urban settlements: evidence from the Nairobi Urban Health and Demographic Surveillance System. J Urban Health. 2011; 88 (Suppl. 2), S200-S218.

35. Campbell MK, Elbourne DR, Altman DG. CONSORT statement: extension to cluster randomised trials. BMJ. 2004; 328, 702-708.

36. Reading R, Harvey I, McLean M. Cluster randomised trials in maternal and child health: implications for power and sample size. Arch Dis Child. 2000; 82, 79-83.

37. Ndugwa RP, Kabiru CW, Cleland J, et al. Adolescent problem behavior in Nairobi's informal settlements: applying problem behavior theory in Sub-Saharan Africa. J Urban Health. 2011; 88 (Suppl. 2), S298-S317.

38. WHO. Infant and Young Child Feeding Counselling: An Integrated Course. 2006. WHO Document Production Services: Geneva.

39. Amendah DD, Mutua MK, Kyobutungi C, Buliva E, Bellows B. Reproductive health voucher program and facility based delivery in informal settlements in Nairobi: a longitudinal analysis. PLoS One. 2013; 8, e80582.

40. World Health Organization. Indicators for assessing breastfeeding practices (reprinted report of an informal meeting 11-12 June), 1991. World Health Organization, Geneva.

41. Kenya National Bureau of Statistics, Ministry of Health, National AIDS Control Council, Kenya Medical Research Institute, National Council for Population and Development. Kenya demographic and health survey 2014: key indicators report, 2015. Kenya National Bureau of Statistics, Ministry of Health, National AIDS Control Council, Kenya Medical Research Institute, National Council for Population and Development, Nairobi.

42. Fotso JC, Ezeh A, Madise N, Ziraba A, Ogollah R. What does access to maternal care mean among the urban poor? Factors associated with use of appropriate maternal health services in the slum settlements of Nairobi, Kenya. Matern Child Health J. 2009; 13, 130-137.

43. Pallas SW, Minhas D, Perez-Escamilla R, Taylor L, Curry L, Bradley EH. Community health workers in low- and middleincome countries: what do we know about scaling up and sustainability? Am J Public Health. 2013; 103, e74-e82.

44. Haider R, Ashworth A, Kabir I, Huttly S. Effect of communitybased peer counsellors on exclusive breastfeeding practices in Dhaka, Bangladesh: a randomised controlled trial. Lancet. 2000; 356, 1643-1647.

45. Wangalwa G, Cudjoe B, Wamalwa D, et al. Effectiveness of Kenya's Community Health Strategy in delivering communitybased maternal and newborn health care in Busia County, Kenya: non-randomized pre-test post test study. Pan Afr Med J. 2012; 13 (Suppl. 1), 12.

46. Aidam BA, Perez-Escamilla R, Lartey A. Lactation counseling increases exclusive breast-feeding rates in Ghana. J Nutr. 2005; 135, 1691-1695.

47. Coutinho SB, de Lira PI, de Carvalho Lima M, Ashworth A. Comparison of the effect of two systems for the promotion of exclusive breastfeeding. Lancet. 2005; 366, 1094-1100. 
48. Bhutta AZ, Lassi ZS, Pariyo G, Huicho L. Global Experience of Community Health Workers for Delivery of Health Related Millennium Development Goals: A Systematic Review, Country Case Studies and Recommendation for Integration into National Health Systems. 2010. World Health Organization: Global Health Workforce Alliance: Geneva.
49. Haines A, Sanders D, Lehmann U, et al. Achieving child survival goals: potential contribution of community health workers. Lancet. 2007; 369, 2121-2131.

50. Lassi ZS, Das JK, Salam RA, Bhutta ZA. Evidence from community level inputs to improve quality of care for maternal and newborn health: interventions and findings. Reprod Health. 2014; 11 (Suppl. 2), S2. 